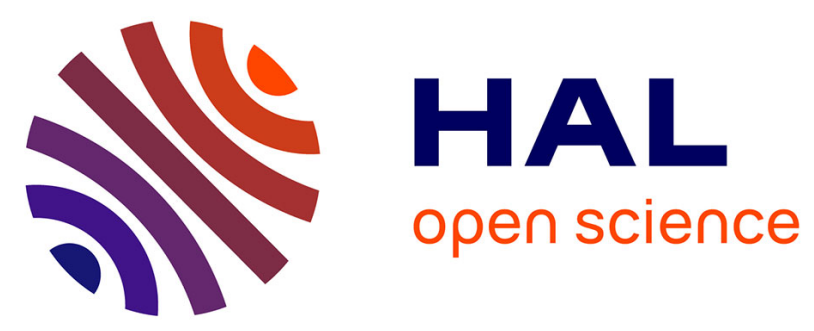

\title{
Orbital Energies and Nuclear Forces in DFT: Interpretation and Validation
}

Rubén Laplaza, Carlos Cárdenas, Patrick Chaquin, Julia Contreras-Garcia, Paul W Ayers

\section{> To cite this version:}

Rubén Laplaza, Carlos Cárdenas, Patrick Chaquin, Julia Contreras-Garcia, Paul W Ayers. Orbital Energies and Nuclear Forces in DFT: Interpretation and Validation. Journal of Computational Chemistry, 2021, 42 (5), pp.334-343. 10.1002/jcc.26459 . hal-03155849

\section{HAL Id: hal-03155849 https://hal.sorbonne-universite.fr/hal-03155849}

Submitted on 2 Mar 2021

HAL is a multi-disciplinary open access archive for the deposit and dissemination of scientific research documents, whether they are published or not. The documents may come from teaching and research institutions in France or abroad, or from public or private research centers.
L'archive ouverte pluridisciplinaire HAL, est destinée au dépôt et à la diffusion de documents scientifiques de niveau recherche, publiés ou non, émanant des établissements d'enseignement et de recherche français ou étrangers, des laboratoires publics ou privés. 


\title{
Orbital Energies and Nuclear Forces in DFT: Interpretation and Validation
}

\author{
Rubén Laplaza ${ }^{1,2^{*}}$, Carlos Cardenas ${ }^{3}$, Patrick Chaquin ${ }^{1}$, \\ Julia Contreras-García ${ }^{1}$, and Paul W. Ayers ${ }^{4}$ \\ ${ }^{1}$ Sorbonne Université, CNRS, Laboratoire de Chimie Théorique, LCT, F \\ 75005 Paris, France \\ ${ }^{2}$ Departamento de Química Física, Universidad de Zaragoza, 50009 \\ Zaragoza, Spain \\ ${ }^{3}$ Departamento de Física, Facultad de Ciencias, Universidad de Chile, \\ Casilla 653, Santiago, Chile and Centro para el desarrollo de la Nanociencias \\ y Nanotecnologia, CEDENNA, Av. Ecuador 3493, Santiago, Chile \\ ${ }^{4}$ Department of Chemistry and Chemical Biology, McMaster University, \\ Hamilton, Ontario, Canada, L8S4M1
}

March 2, 2021

\begin{abstract}
The bonding and antibonding character of individual Molecular Orbitals has been previously shown to be related to their orbital energy derivatives with respect to nuclear coordinates, known as Dynamical Orbital Forces. Albeit usually derived from Koopmans' theorem, in this work we show a more general derivation from conceptual DFT, which justifies application in a broader context. The consistency of the approach is validated numerically for valence orbitals in Kohn-Sham DFT. Then, we illustrate its usefulness by showcasing applications in aromatic and antiaromatic systems and in excited state chemistry. Overall, Dynamical Orbital Forces can be used to interpret the results of routine ab initio calculations, be it wavefunction or density based, in terms of forces and occupations.
\end{abstract}

Keywords: Density Functional Theory, Dynamic Orbital Forces, Nuclear Fukui Function, Nuclear Forces, Conceptual Density Functional Theory 


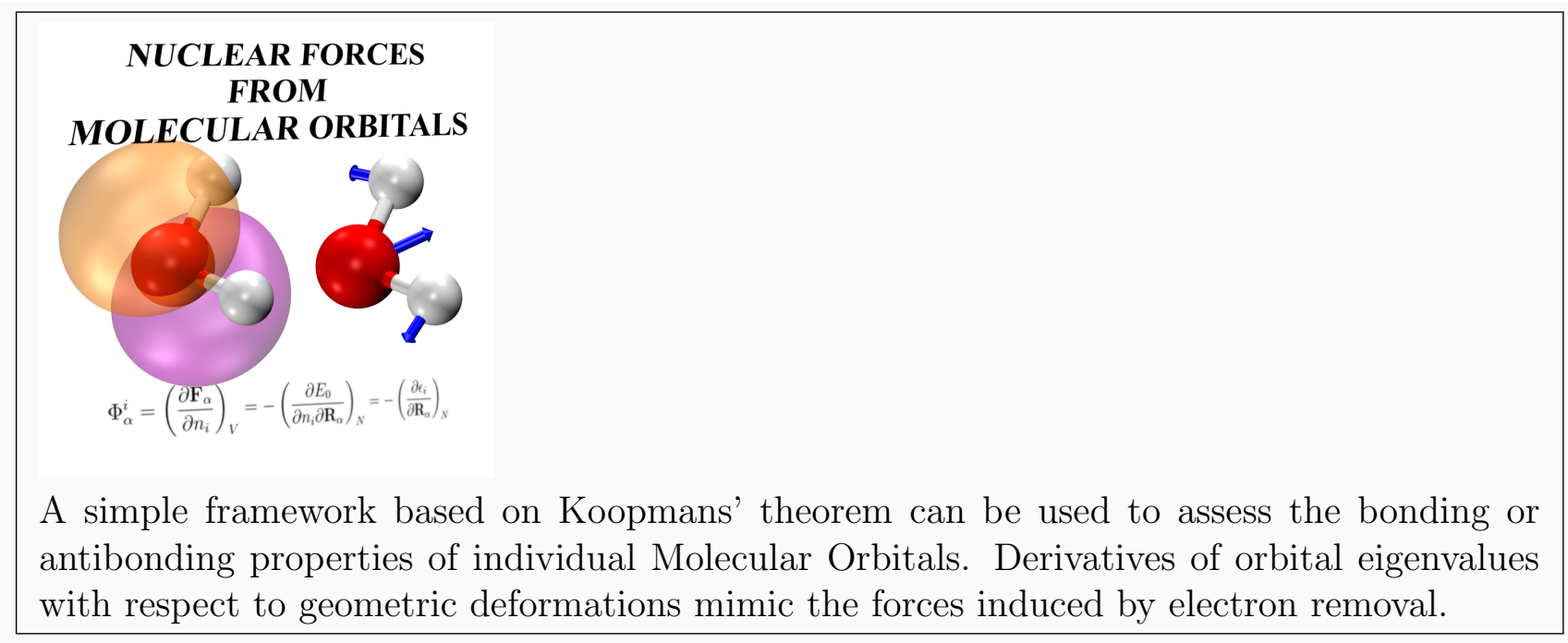




\section{INTRODUCTION}

Molecular Orbital Theory (MOT) constitutes the main theoretical framework of chemistry, yet individual Molecular Orbitals (MOs), especially as the complexity of the system under consideration increases, are difficult to characterize in a quantitative way. While in simple systems a character can be assigned to individual MOs with ease, cases with sophisticated hybridization character and low symmetry are hard to classify: the weight and effect of non-local MOs on individual bonds or interactions is highly problematic.

Orbital forces have been used in several contexts for the individual characterization of MOs in terms of bonding or antibonding character with respect to individual bonds. ${ }^{1-5}$ This technique stems from the ideas of Tal and Katriel, ${ }^{6}$ which are based on the famed Koopmans' theorem. The main idea is captured in Eq. 1, which is simply an extension of the theorem in which the ground state energy of a system $\left(E^{0}\right)$ is related to the energy of the corresponding $i$ th cation, in which an electron has been removed from orbital $i\left(E_{i}^{+}\right)$that has, in the ground state calculation, an associated eigenvalue $\epsilon_{i}$ and an ionization energy $I_{i}$ :

$$
\epsilon_{i} \approx E^{0}-E_{i}^{+}=I_{i}
$$

Koopmans' theorem is exact in monodeterminantal Hartree-Fock Theory (HFT) as long as orbital relaxation is neglected, which is to say the MOs of the cation are the same as in the neutral species. This is generally untrue, which introduces a significant error and thus demands the approximation sign in Eq. 1. Fortunately, the major caveat of HFT, which is it does not account for instantaneous electron correlation, seems to partially cancel with the error derived from orbital relaxation. Therefore, through error compensation, Koopmans' theorem provides good vertical excitation energies for many molecules. ${ }^{7}$

By taking derivatives of Eq. 1 with respect to a nuclear coordinate $\mathbf{R}$ in a BornOppenheimer context, we obtain the following expression (Eq. 2):

$$
\frac{\partial \epsilon_{i}}{\partial \mathbf{R}} \approx \frac{\partial E^{0}}{\partial \mathbf{R}}-\frac{\partial E_{i}^{+}}{\partial \mathbf{R}}
$$

that further simplifies to Eq. 3 if the fixed, equilibrium ground state geometry is considered $\left(\mathbf{R}_{\mathbf{e q}}\right)$ and thus $\partial E^{0} / \partial \mathbf{R}_{\mathbf{e q}}=0$ for any internal coordinate: 


$$
\frac{\partial E_{i}^{+}}{\partial \mathbf{R}_{\mathbf{e q}}} \approx-\frac{\partial \epsilon_{i}}{\partial \mathbf{R}_{\mathbf{e q}}}
$$

Hence, as seen in Eq. 3, by analyzing the derivatives of MO energies (i.e. eigenvalues) in the ground state species with respect to a nuclear displacement (second term), one can in principle predict the forces induced on the nuclei (first term) upon removal of an electron from the corresponding $i$ th orbital. This can be used to classify MOs into bonding or antibonding with respect to any coordinate deformation, such as pairwise elongation or shortening corresponding to a bond.

The force term derived from the MO eigenvalue $\epsilon_{i}$ that appears in the right side of Eq. 3 is what has been called a Dynamic Orbital Force (DOF). A set of $i$ DOFs can be defined, one per MO in the system. In principle, according to Eq. 3, such quantities let us link cationic states and ground state properties.

Unfortunately, even when Eq. 3 holds, the predicted gradients have limited usefulness due to the inaccuracy of HFT, which does not properly account for electron correlation. Therefore, even if the equality is exact, the left hand term in Eq. 3 is highly questionable. Thus, it would be desirable to extend the aforementioned framework to Kohn-Sham (KS) Density Functional Theory (DFT), which is significantly more accurate and widely used. However, a new source of error is added, namely the accuracy of the Density Functional Approximation (DFA) to the exchange-correlation functional. Consequently, the fortuitous error compensation that enables Eq. 1 in HFT in the first place might not be valid at all in DFT. On the other hand, the errors due to the lack of electron correlation treatment may in principle disappear if the exact functional is considered. There is a wealth of literature in this regard, both from the theoretical and the empirical points of view, ${ }^{8-12}$ but Koopmans' theorem and its consequences are routinely used in a KS-DFT context, using KS orbital energies without much questioning.

In this paper we justify and foster the usage and interpretation of DOFs calculated from KS-DFT molecular orbitals. A unified theoretical background will be provided. Then, the validity of the main assumptions will be tested empirically, and finally some example applications will be presented. 


\section{METHODOLOGY}

All calculations were first performed using the PSI4 v.1.1 package, ${ }^{13}$ using the Maximum Overlap Method ${ }^{14,15}$ for all $\Delta$-SCF excited state cation calculations. DOF calculations were performed with an in-house finite differences code using the helpful Python framework of HORTON 2.1, ${ }^{16}$ PySCF $^{17}$ and libcint. ${ }^{18}$ All DFAs were used as defined by the Libxc library $^{19}$ as PBE, PBE0 and B3LYPG. Graphical representations were produced with the VMD program. ${ }^{20}$

\section{RESULTS AND DISCUSSION}

The structure of this paper is the following: first, a unified derivation for Dynamic Orbital Forces will be given in the DFT framework, closely related to the work of Averill and Painter, ${ }^{21}$ and possible interpretations will be examined. Then, both the right and left-side terms in Eq. 3, the DOFs and the nuclear gradients of the $i$ th cations, will be computed using both HFT and DFT. By comparison between the two terms, the validity of Koopmans' theorem and its extension to DFT in this particular regard will be ascertained and condensed to a simple list of do's and and don'ts. Finally, some examples of applications will be presented in simple molecules.

\section{Unified derivation and interpretation}

The concept and interpretation of DOFs can be derived from the conceptual DFT framework, thus independently from Eq. 1. In fact, Nuclear Fukui Functions (NFF) as defined by Cohen and Ganduglia-Pirovano, ${ }^{22,23}$ can be seen as a particular case of DOFs. NFFs, $\Phi_{\alpha}$, measure the variation of the force experienced by a nucleus $\alpha$ upon variation of the number of electrons $N$ with constant external potential $v$. Such descriptor is analogous to Fukui functions, but focused in nuclear effects. NFFs can be expressed as the opposite of the variation of the molecular electronic chemical potential $\mu$ upon variation of the nuclear coordinates $\mathbf{R}_{\alpha}$ with a constant number of electrons $N$, as shown in Eq. $4 .^{24}$ 


$$
\Phi_{\alpha}=\left(\frac{\partial \mathbf{F}_{\alpha}}{\partial N}\right)_{v}=-\left(\frac{\partial \mu}{\partial \mathbf{R}_{\alpha}}\right)_{N}
$$

NFFs have previously been used for the rationalization of the Jahn-Teller effect and the effect of electron addition upon equilibrium bond lengths. ${ }^{25,26}$

If $\mu$ in Eq. 4 is expressed as $1 / 2\left(\epsilon_{H O M O}-\epsilon_{L U M O}\right)$ it becomes apparent that $\Phi$ arises from subtracting the forces arising from the LUMO from the forces arising from the HOMO, which in the ground state equilibrium geometry further simplifies as in Eq. 5, where the rightmost term assumes Eq. 3 holds. In the equilibrium geometry, NFFs reflect whether the forces exerted on nuclei as a result of electron addition or removal (in the LUMO and HOMO MOs respectively) dominate. Simply put, it is able to predict how the forces upon nuclei will change upon globally changing $N$, which is the same as predicting whether electron acception or donation will become more favorable upon nuclear displacement. For the energies $E_{\text {HOMO }}^{+}$ and $E_{L U M O}^{-}$of the HOMO cation and the LUMO anion respectively, we may write (Eq. 5):

$$
\Phi_{\alpha, \mathbf{e q}} \approx \frac{1}{2}\left(\frac{\partial \epsilon_{L U M O}}{\partial \mathbf{R}_{\alpha, e q}}-\frac{\partial \epsilon_{H O M O}}{\partial \mathbf{R}_{\alpha, e q}}\right) \approx \frac{1}{2}\left(\frac{\partial E_{H O M O}^{+}}{\partial \mathbf{R}_{\alpha, e q}}-\frac{\partial E_{L U M O}^{-}}{\partial \mathbf{R}_{\alpha, e q}}\right)
$$

The same concept can be extrapolated to construct orbital NFFs $\Phi_{\alpha}^{i}$ in which the derivative of the force is taken with respect of the individual occupation $n_{i}$ of the $i$ th MO. As per Janak's theorem, ${ }^{27}$ such orbital NFFs are equivalent to DOFs in the equilibrium geometry of the ground state (Eq. 6), as previously derived from a different pathway $^{21}$ :

$$
\Phi_{\alpha}^{i}=\left(\frac{\partial \mathbf{F}_{\alpha}}{\partial n_{i}}\right)_{v}=-\left(\frac{\partial E_{0}}{\partial n_{i} \partial \mathbf{R}_{\alpha}}\right)=-\left(\frac{\partial \epsilon_{i}}{\partial \mathbf{R}_{\alpha}}\right)_{N}
$$

Note that the transformation implicitly owes to the derivatives of the ground state energy with respect to the occupation numbers. ${ }^{28}$ Thus, DOFs, which are orbital NFFs in the ground state equilibrium geometry, determine whether decreasing occupation in that specific MO brings certain atoms closer or pushes them apart. The concept is faithful to the core principles and interpretations of MOT: the bonding or antibonding character of any individual or subgroup or MOs can be unequivocally ascertained by using the complete set of DOFs. When depicted as a set of atomic displacement vectors departing from the equilibrium ground state geometry, $\Phi_{\alpha}^{i}$ represent the forces that arise whenever an electron is 
subtracted from the $i$ th $\mathrm{MO}$, while $-\Phi_{\alpha}^{i}$ represent the predicted forces for when an electron is added to the $i$ th MO.

\section{Static and dynamic forces}

By assuming Hellmann-Feynman forces are sufficient in the previous expression, we can rewrite $\Phi_{\alpha}^{i}$ as (Eq. 7):

$$
\Phi_{\alpha}^{i}=Z_{\alpha} \int\left(\frac{\partial \rho(\mathbf{r})}{\partial n_{i}}\right)_{v} \frac{\left(\mathbf{r}-\mathbf{R}_{\alpha}\right)}{\left|\mathbf{r}-\mathbf{R}_{\alpha}\right|^{3}} d \mathbf{r}
$$

in which $\mathbf{r}, \mathbf{R}$ are electronic and nuclear coordinates respectively, $Z_{\alpha}$ is the nuclear charge of nuclei $\alpha$ and $\rho(r)$ is the electron density. The derivative in the integral in Equation 7 can be expressed as the sum of two terms (Eq. 8),

$$
\left(\frac{\partial \rho(\mathbf{r})}{\partial n_{i}}\right)_{v}=\left(\frac{\partial \sum_{j}^{N} n_{j}\left|\psi_{j}(\mathbf{r})\right|^{2}}{\partial n_{i}}\right)_{v}=\left|\psi_{i}(\mathbf{r})\right|^{2}+\sum_{j}^{N} \frac{n_{j} \partial\left|\psi_{j}(\mathbf{r})\right|^{2}}{\partial n_{i}}
$$

in which $\psi_{j}$ are the different MOs in the Kohn-Sham wave function, including the $i$ th MO $\psi_{i}$. The $\left|\psi_{i}(\mathbf{r})\right|^{2}$ term in the rightmost side of Eq. 8 represents the change in occupation of the MO under consideration while the second term represents orbital relaxation phenomena. This orbital relaxation term is often very small, and thus is usually neglected. The underlying assumption is that MOs do not change significantly whenever the occupation of a different MO changes, which is a weaker constraint than the one implied by Koopmans' theorem, in which all MOs remain unaltered. By following this assumption the following expression is recovered (Eq. 9), where the $\psi$ subscript indicates that the orbitals remain unaltered:

$$
\left(\Phi_{\alpha}^{i}\right)_{\psi}=Z_{\alpha} \int \frac{\left|\psi_{i}(\mathbf{r})\right|^{2}}{\left|\mathbf{r}-\mathbf{R}_{\alpha}\right|^{3}}\left(\mathbf{r}-\mathbf{R}_{\alpha}\right) d \mathbf{r}
$$

With the removal of the orbital relaxation term, Eq. 9 reveals the foundation of DOFs, which are simply the forces arising from the electrostatic interaction between the density in a particular $\mathrm{MO} \psi_{i}$ and a nuclei $\alpha$, or, in other words, the $i$ th $\mathrm{MO}$ contribution to the Hellmann-Feynman force on that nuclei, or static force by analogy.

However, the inclusion of orbital relaxation effects is not always negligible. Recalling the following equalities given by the construction of the Hamiltonian (Eqs.10 and 11) and 
the derivatives of the Fock operator, where $T$ is the electronic kinetic energy, $U_{N e}$ is the nuclear-electron attraction energy, $V_{N N}$ accounts for the nuclear-nuclear repulsion energy, $W_{e e}$ is the (approximate) electron-electron interaction energy, and Pulay forces are ignored (analogous results are obtained using a Kohn-Sham formulation in this regard):

$$
\begin{gathered}
E_{0}=T+U_{N e}+V_{N N}+W_{e e}=\sum_{i}^{N} \epsilon_{i}-W_{e e}+V_{N N} \\
\sum_{i}^{N} \frac{\partial \epsilon_{i}}{\partial \mathbf{R}}=\frac{\partial E_{0}}{\partial \mathbf{R}}+\frac{\partial W_{e e}}{\partial \mathbf{R}}-\frac{\partial V_{N N}}{\partial \mathbf{R}}=\frac{\partial T}{\partial \mathbf{R}}+\frac{\partial U_{N e}}{\partial \mathbf{R}}+2 \frac{\partial W_{e e}}{\partial \mathbf{R}}
\end{gathered}
$$

As given by Eq. 9, note that the sum of all static forces is analogous to $\partial U_{N e} / \partial \mathbf{R}$ (Eq. $12)$ :

$$
\sum_{i}^{N}\left(\frac{\partial \epsilon_{i}}{\partial \mathbf{R}}\right)_{\psi}=-\sum_{i}^{N}\left(\Phi^{i}\right)_{\psi}=\frac{\partial U_{N e}}{\partial \mathbf{R}}
$$

Therefore, the application of Eq. 9 neglects the derivative of (twice) the effective electronelectron term $W_{e e}$ and the one electron kinetic energy $T$. Thus, it assumes that the sum of those derivatives is negligible with respect to the derivative of the nuclear-electron term $U_{N e}$.

Generally, the neglected terms can be presumed to be small and the approximation is valid. However, within the Born-Oppenheimer approximation, the divergence term will increase enormously whenever, over a particular coordinate $\mathbf{R}$, electronic reorganization is more energetically relevant than nuclear displacement in the potential energy surface. This is particularly relevant if additions or subtractions of DOFs are to be performed, and specially in the study of chemical reactivity. ${ }^{29}$ The quantification of the total divergence term is easily available as Eq. 13 in HFT, or Eq. 14 in KS-DFT, where $T_{s}$ is the kinetic energy of the KS system, $E_{H}$ is the Hartree energy and $V_{x c}$ is the exchange-correlation potential:

$$
\begin{gathered}
\sum_{i}^{N}\left[\Phi^{i}-\left(\Phi^{i}\right)_{\psi}\right]=\frac{\partial T}{\partial \mathbf{R}}+2 \frac{\partial W_{e e}}{\partial \mathbf{R}} \\
\sum_{i}^{N}\left[\Phi^{i}-\left(\Phi^{i}\right)_{\psi}\right]=\frac{\partial T_{s}}{\partial \mathbf{R}}+2 \frac{\partial E_{H}}{\partial \mathbf{R}}+2 \int \rho(\mathbf{r}) V_{x c}(\mathbf{r}) d \mathbf{r}
\end{gathered}
$$


Note that while the total difference is expected to be small, this is not necessarily true for individual $i$ th contributions. Therefore, in this work DOFs will be computed including the orbital relaxation term, as the true derivatives of the MO eigenvalues, and always from the equilibrium ground state geometry. Formally, the inclusion or neglect of the orbital relaxation term in the integrand of Eq. 7 is analogous to the difference between Fukui functions and classic Frontier Molecular Orbital (FMO) theory. Therefore, DOFs need not to be confused with Hellmann-Feynman forces or static forces.

Summarizing, a set of DOFs $\Phi^{i}$, acting on each nuclei $\alpha$, can be constructed per each MO $i$ of a molecular system. If taken around the equilibrium geometry, $\mathbf{R}=\mathbf{R}_{\mathbf{e q}}$, DOFs are expected to satisfy

$$
\Phi^{i}=-\frac{\partial \epsilon_{i}}{\partial \mathbf{R}_{\mathbf{e q}}}=\left(\frac{\partial \mathbf{F}}{\partial n_{i}}\right)_{v} \approx \frac{\partial E_{i}^{+}}{\partial \mathbf{R}_{\mathbf{e q}}}
$$

in which the approximate equality in Eq. 15 is exact if Koopmans' theorem holds. In the domain of chemical bonding, analyzing or representing $\Phi_{\alpha}^{i}$ predicts the distortion in the molecular geometry that happens when an electron is removed from the $i$ th MO of the ground state, and $-\Phi_{\alpha}^{i}$ works similarly for whenever an electron is added to the $i$ th MO.

\section{Accuracy of DOFs in DFT}

This section focuses on verifying that DOFs calculated from KS MOs are valid. Recall that there are two sources of error at hand here: orbital relaxation in the electronically relaxed cation, and the accuracy of the exchange-correlation functional.

The rightmost and leftmost terms of Eq. 3 have been calculated for the water molecule using four different methodologies (HFT, PBE, PBE0 and B3LYP) and nine different basis sets, namely cc-pVDZ, cc-pVTZ, cc-pVQZ and the analogous triads including diffuse (aug-)

and core-valence correlation $(c)$ functions. ${ }^{30-32}$ For reference, the canonical MOs of water are represented in Figure 1, and labeled upwards from $i=1$ to $N / 2=5$, such that $i=5$ for the HOMO. Recall that the $\partial E_{i}^{+} / \partial \mathbf{R}_{\mathbf{e q}}$ terms have been calculated using the appropriate electronically relaxed $i$ th cations. 


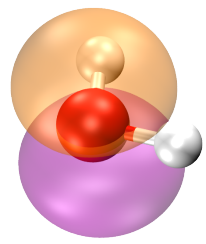

5

$1 b_{1}$

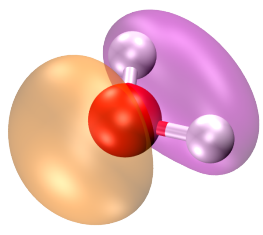

4

$3 a_{1}$

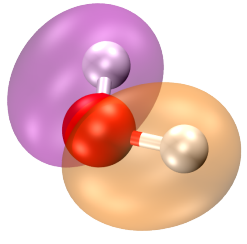

3

$1 b_{2}$

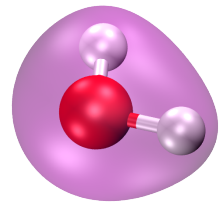

2

$2 a_{1}$

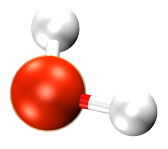

1

$1 a_{1}$

Figure 1: Isosurface representation of the canonical MOs of water and their respective symmetry labels.

To compare the gradient for each atom in the relaxed cations and the corresponding $\Phi_{\alpha}^{i}$ (Eq. 3), the Cartesian norm $\left(L^{2}\right)$ was used, measuring the distance between each pair of vectors with the same center (see Fig. 2). Both the magnitude and the alignment between the gradient vectors are implicitly considered in $L^{2}$, however the alignment is more relevant and thus will be measured explicitly as the cosine of the angle $\theta$ between vector pairs.

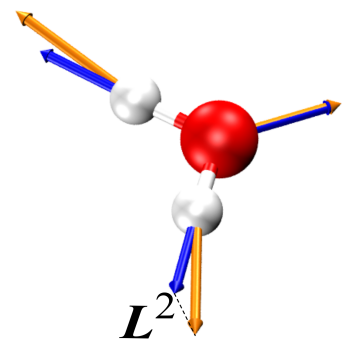

Figure 2: $L^{2}$ indicated as a dotted black line for the HOMO $\left(1 b_{1}\right)$ orbital of water: cartesian distance between $\partial E_{5}^{+} / \partial \mathbf{R}_{\mathbf{e q}}$ (orange) and $-\partial \epsilon_{5} / \partial \mathbf{R}_{\mathbf{e q}}$ (blue). Note that the $x$ axis is perpendicular to the plane of the molecule.

The results of this comparison can be interpreted on two levels. First, if the alignment $(\cos (\theta))$ between the gradients in the $i$ th cation, where the electron has been removed from the $i$ th $\mathrm{MO}$, and the set of $\Phi_{\alpha}^{i}$, is always close to 1.0, all the qualitative information is properly captured. If, furthermore, the $L^{2}$ norms between vector pairs are small, results are quantitative and Koopmans' theorem holds.

First of all, it is important to check that the alignment between the gradients of the cationic species (first three columns of Table 1) are aligned independently of the method. 
This means that there is a similar qualitative effect that is shared between HF, PBE, PBE0 and B3LYP. Therefore, it can be assumed that all discrepancies are due to Koopmans' failure: differences in alignment arise from $\Phi_{\alpha}^{i}$, not from different descriptions of the cations.

A detailed comparison for the HF/aug-cc-pVQZ level of theory is shown in Table 1. In this case, the qualitative aspect (i.e. the alignment) is accurate in all cases except for the lowest lying core MO 1 , where $\cos (\theta) \approx-1$ (i.e. counteralignment). Examining Table 1 reveals that MOs 2 and 3, HOMO-3 and HOMO-2 respectively, have the highest gradient values, which aim to unbind the molecule when an electron is removed from them, confirming their bonding character. MO 5, the HOMO, is fundamentally non-bonding (i.e. gradients are very small in all directions), and the HOMO-1 is very weakly antibonding. All these are in perfect agreement with classical MO theory: the HOMO orbital in water is fundamentally non-bonding, the HOMO-1 has some antibonding character resulting from the $2 \mathrm{~s}$ orbital of oxygen, and the HOMO-2 and HOMO-3 effectively bind the molecule together. Note that, while we are discussing the role of individual orbitals in the stability of the molecule as a whole, it is possible to project forces on internuclear axes to assess their roles in individual bonds as well. Both the cationic gradients and the values of $\Phi_{\alpha}^{i}$ give relevant information, which is this case is in perfect interpretative agreement. 


\begin{tabular}{c|cccc|rrrr|r}
$\alpha$ & $i$ & $x$ & $y$ & $z$ & $i$ & $x$ & $y$ & $z$ & $\cos (\theta)$ \\
\hline $\mathrm{O}$ & & 0.0000 & 0.0000 & -0.0622 & & 0.0000 & 0.0000 & 0.2460 & -1.0000 \\
$\mathrm{H}$ & $\frac{\partial E_{1}^{+}}{\partial \mathbf{R}_{\alpha}}$ & 0.0000 & -0.0913 & 0.0311 & $\Phi_{\alpha}^{1}$ & 0.0000 & 0.1738 & -0.1230 & -0.9589 \\
$\mathrm{H}$ & & 0.0000 & 0.0913 & 0.0311 & & 0.0000 & -0.1738 & -0.1230 & -0.9589 \\
\hline $\mathrm{O}$ & & 0.0000 & 0.0000 & 0.2822 & & 0.0000 & 0.0000 & 0.3950 & 1.0000 \\
$\mathrm{H}$ & $\frac{\partial E_{2}^{+}}{\partial \mathbf{R}_{\alpha}}$ & 0.0000 & 0.2323 & -0.1411 & $\Phi_{\alpha}^{2}$ & 0.0000 & 0.2753 & -0.1975 & 0.9971 \\
$\mathrm{H}$ & & 0.0000 & -0.2323 & -0.1411 & & 0.0000 & -0.2753 & -0.1975 & 0.9971 \\
\hline $\mathrm{O}$ & & 0.0000 & 0.0000 & 0.5562 & & 0.0000 & 0.0000 & 0.2668 & 1.0000 \\
$\mathrm{H}$ & $\frac{\partial E_{3}^{+}}{\partial \mathbf{R}_{\alpha}}$ & 0.0000 & 0.1455 & -0.2781 & $\Phi_{\alpha}^{3}$ & 0.0000 & 0.0737 & -0.1334 & 0.9997 \\
$\mathrm{H}$ & & 0.0000 & -0.1455 & -0.2781 & & 0.0000 & -0.0737 & -0.1334 & 0.9997 \\
\hline $\mathrm{O}$ & & 0.0000 & 0.0000 & -0.0556 & & 0.0000 & 0.0000 & -0.0484 & 1.0000 \\
$\mathrm{H}$ & $\frac{\partial E_{4}^{+}}{\partial \mathbf{R}_{\alpha}}$ & 0.0000 & 0.1376 & 0.0278 & $\Phi_{\alpha}^{4}$ & 0.0000 & 0.0780 & 0.0242 & 0.9949 \\
$\mathrm{H}$ & & 0.0000 & -0.1376 & 0.0278 & & 0.0000 & -0.0780 & 0.0242 & 0.9949 \\
\hline $\mathrm{O}$ & & 0.0000 & 0.0000 & 0.0613 & & 0.0000 & 0.0000 & 0.0372 & 1.0000 \\
$\mathrm{H}$ & $\frac{\partial E_{5}^{+}}{\partial \mathbf{R}_{\alpha}}$ & 0.0000 & 0.0380 & -0.0307 & $\Phi_{\alpha}^{5}$ & 0.0000 & 0.0410 & -0.0186 & 0.9683 \\
$\mathrm{H}$ & 0.0000 & -0.0380 & -0.0307 & & 0.0000 & -0.0410 & -0.0186 & 0.9683
\end{tabular}

Table 1: Nuclear gradients for the cationic species and corresponding $\Phi_{\alpha}^{i}$, per nuclei $\alpha$ per cartesian direction $(\mathrm{x}, \mathrm{y}, \mathrm{z})$ in atomic units, calculated at the HF/aug-cc-pVQZ level. Cosines of the angle between each pair $(\theta)$ are shown right.

Table 2 offers the same information compared in terms of $L^{2}$ and averaged over $\alpha$ and $i$. The average $L^{2}$ norm over $\alpha$ has been termed $\bar{L}_{\alpha}^{2}$, while averages over individual MOs are expressed as $\bar{L}_{i}^{2}$. Note how the conclusion that the agreement is good except for the core MO, as seen in Table 1 , is now conveyed by the average value of $i=1$, or $\bar{L}_{1}^{2}$, in a much more compact way.

Plotting $\bar{L}_{i}^{2}$ for each method and basis set (Fig. 3) corroborates that DOFs from valence MOs are generally in good agreement $\left(\bar{L}_{i}^{2} \approx 0\right)$, especially with large augmented basis sets. The DOFs associated with MO $1, \Phi^{1}$, which concern essentially the core orbital of the oxygen atom, are in significantly worse agreement than the others, and therefore have much larger $\bar{L}_{1}^{2}$ values (red bars). In this regard, DFT (Fig. 3B-D) is significantly worse than HFT (Fig. 3A). Koopmans' theorem does not seem to hold for the core orbital in general, which 


\begin{tabular}{|c|c|c|c|c|c|c|}
\hline & 5 & 4 & 3 & 2 & 1 & $\bar{L}_{\alpha}^{2}$ \\
\hline $\mathrm{O}$ & 0.0241 & 0.0072 & 0.2894 & 0.1127 & 0.3082 & 0.1483 \\
\hline $\mathrm{H}$ & 0.0124 & 0.0597 & 0.1615 & 0.0709 & 0.3067 & 0.1222 \\
\hline $\mathrm{H}$ & 0.0124 & 0.0597 & 0.1615 & 0.0709 & 0.3067 & 0.1222 \\
\hline $\bar{L}_{i}^{2}$ & 0.0163 & 0.0422 & 0.2041 & 0.0848 & 0.3072 & 0.1309 \\
\hline
\end{tabular}

Table 2: $L^{2}$ distances (a.u.) between matching pairs of cationic nuclear gradients and $\Phi_{\alpha}^{i}$, calculated at the HF/aug-cc-pVQZ level. All data in atomic units.

can be attributed to the orbital relaxation effects that the wave function may experience when an electron is removed from such a stable MO. The removal of core density induces a drastic rearrangement of the other orbitals, which can not be accounted for starting from the ground state neutral MOs. It can also be argued that decontracted basis sets are necessary for a proper treatment of core holes. However, in any case, we expect the derivatives or core orbitals to be small in relative terms and uninteresting from an interpretative point of view. In all other cases a qualitative and often quantitative agreement is found. 

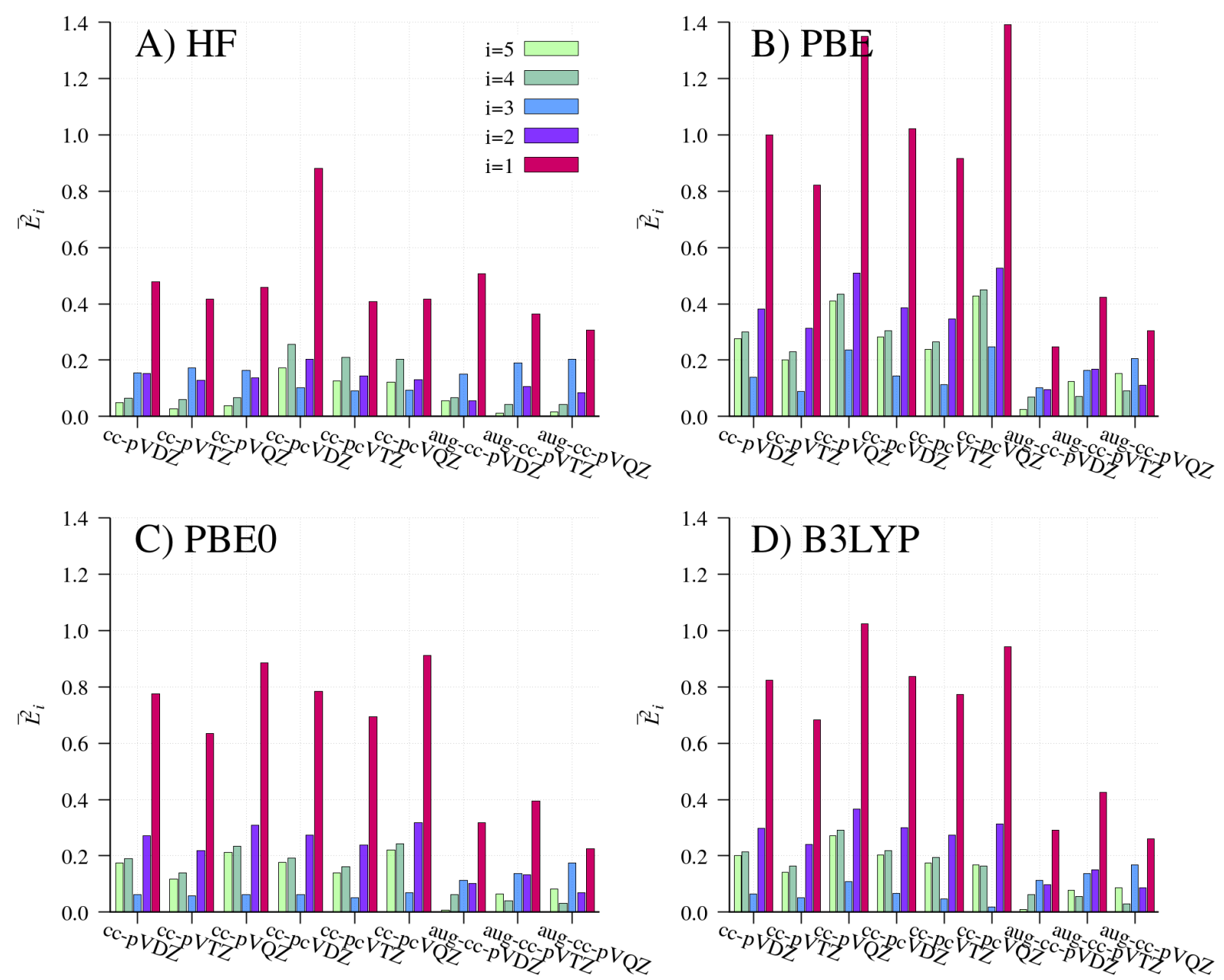

Figure 3: $\bar{L}_{i}^{2}$ (a.u.) per MO per basis for each of the four methods considered in the water molecule: A) HF, B) PBE, C) PBE0, D) B3LYP.

The average norm over both $i$ and $\alpha$ can be used as a global descriptor of the agreement that we shall denote simply $\bar{L}^{2}$. Figure 4 shows the values of $\bar{L}^{2}$ for all the combinations considered in the water molecule. Detailed data can be found in the Supporting Information.

It can be seen that correspondence in HF calculations improves slightly with larger basis sets, while all considered DFAs seem to improve greatly when including diffuse functions but not necessarily with basis set size or core-valence functions (Fig. 4). In particular, corevalence functions seem to alter the magnitude of cationic gradients even if the result of $\Phi_{\alpha}^{i}$ is relatively similar with and without such functions. Diffuse functions, on the contrary, barely affect cationic gradients, but improve the DOF set noticeably. When a suitable basis set is 
used, DFT results are similar to the HF ones in terms of $L^{2}$ distances, and hence as valid as HFT-derived values (Fig. 3, Fig. 4). Noticeably, the aug-cc-pVDZ basis set is extremely successful in spite of its relatively small size. Hybrid DFAs seem to outperform the pure $\mathrm{PBE}$ in this regard, and offer better agreements in general, which is reasonable considering that they are closer to the HFT Hamiltonian.

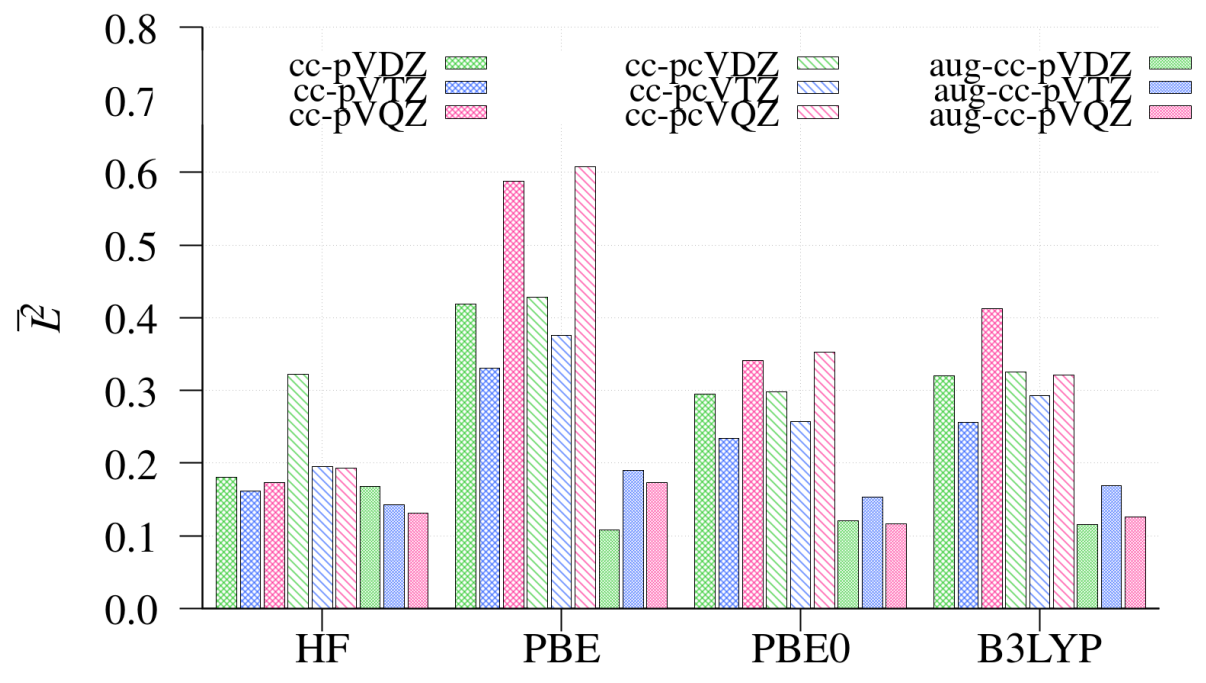

Figure 4: $\bar{L}^{2}$ distance (a.u.) per method per basis set in the water molecule.

Nevertheless, it should be noted that this assessment Sconcerns the degree of agreement between the right and left sides of Equation 3, and not the absolute quality of $\Phi_{\alpha}^{i}$. Therefore, it can be said based on the results summarized in Figures 1 and 2 that DOFs are in agreement with the corresponding cation gradients calculated at the same level of theory, in both HFT and DFT as long as the basis set is appropriate. The sole exception are core orbitals, which are not very interesting from the bonding or reactive point of view anyway.

Following these two notions, an analogous comparison was performed on the ethene molecule. In this case, only the large aug-cc-pVQZ basis set was used, and only the most relevant MOs, shown in Figure 5, were considered. 


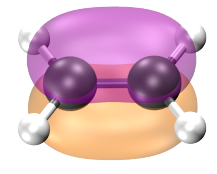

8

$2 b_{1 u}$

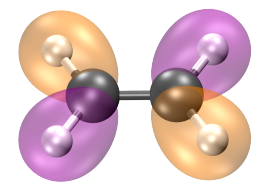

7

$1 b_{2 u}$

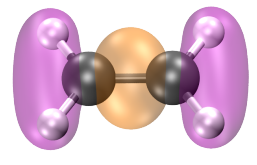

6

$3 a_{g}$

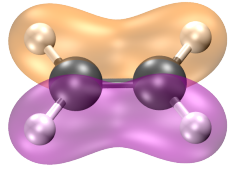

5

$1 b_{3 g}$

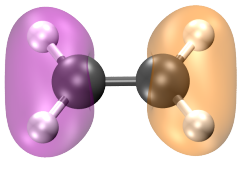

4

$1 b_{3 u}$

Figure 5: Isosurface representation of the five highest energy occupied canonical MOs of ethene and their respective symmetry labels.

The obtained results are remarkably good in both HFT and DFT, summarized in Fig. 6 in terms of $\bar{L}_{i}^{2}$. Note the significantly reduced scale in the $y$-axis, compared to Fig. 3. The alignment of the relevant gradient components is excellent in all cases, even though HF seems to generally outperform DFT once more. Hybrid DFAs (PBE0, B3LYP) have slightly better agreement than PBE, but in all cases the qualitative behavior is well-captured and the norm of all vectors is not significantly different. In this case, for the HOMO, the results are fundamentally quantitative, with $\bar{L}_{i}^{2}$ norms very near zero. In all other cases the general trend is captured and the relative magnitude among the different nuclear forces and MOs is reproduced. In other words, DOFs can be calculated through Koopmans' theorem at inexpensive levels of calculation since the difference between $\partial E_{i}^{+} / \partial \mathbf{R}_{\mathbf{e q}}$ and $-\partial \epsilon_{i} / \partial \mathbf{R}_{\mathbf{e q}}$ is very small for valence orbitals. 

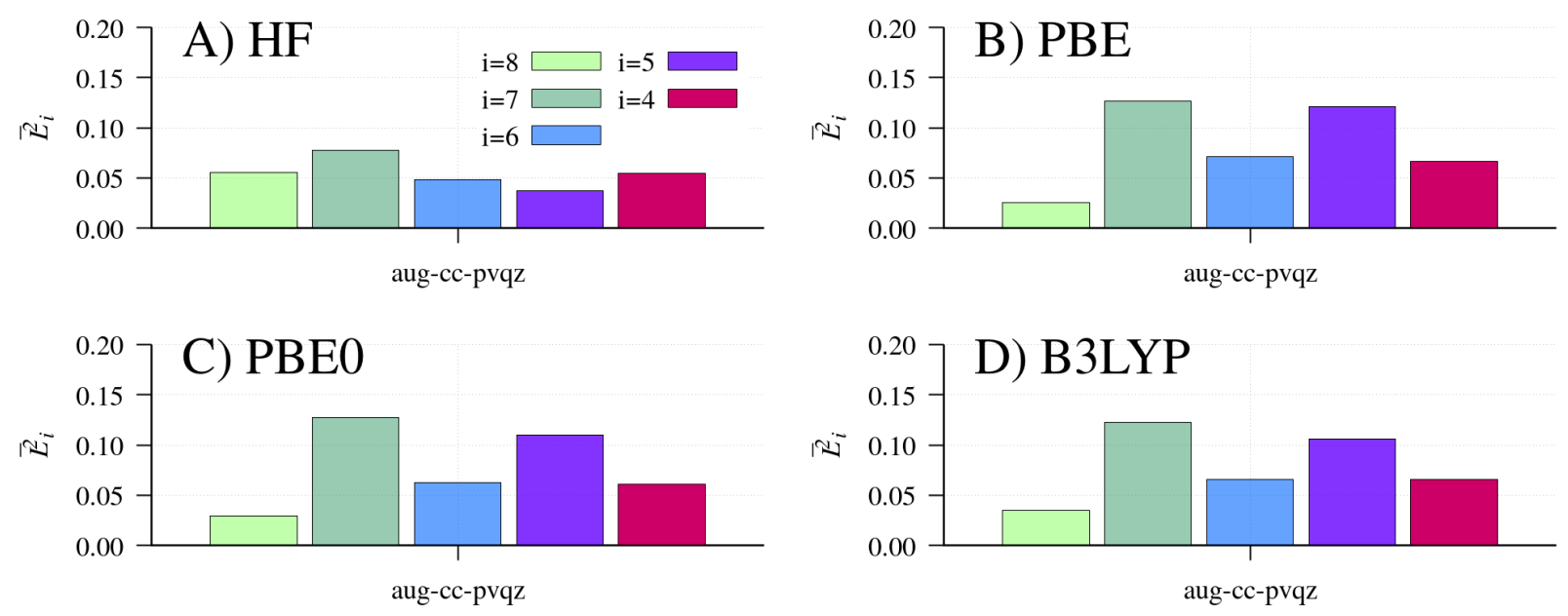

Figure 6: $\bar{L}_{i}^{2}$ (a.u.) per MO for each of the four methods considered in the ethene molecule: A) HF, B) PBE, C) PBE0, D) B3LYP.

The direction of the set of $\Phi^{i}$ matches well-established chemical insight: MO 8, the HOMO, is bonding for the $\mathrm{C}-\mathrm{C}$ bond, while MO 7 is clearly anti-bonding. MO 6 is bonding again, with similar strength as the HOMO. MO 5 concerns mostly $\mathrm{C}-\mathrm{H}$ bonds, but is mostly non-bonding for the $\mathrm{C}-\mathrm{C}$ bond, and finally $\mathrm{MO} 4$, HOMO-4, is strongly $\mathrm{C}-\mathrm{C}$ antibonding, as expected. The complete dataset is available in the SI, and a vector schematic representation of $\Phi_{\alpha}^{i}$ is given for the HF case in Figure 7. For proper interpretation, recall that the length and direction of the vectors that represent $\Phi_{\alpha}^{i}$ match the forces that arise on nuclei with electron removal from the $i$ th $\mathrm{MO}$. 


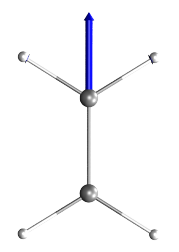

8

$2 b_{1 u}$

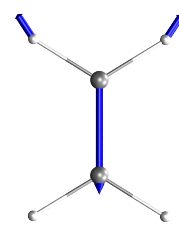

7

$1 b_{2 u}$

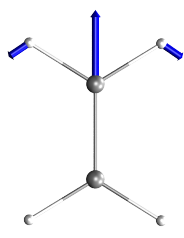

6

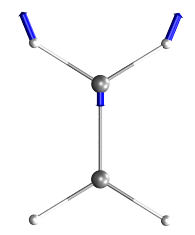

5

$1 b_{3 g}$

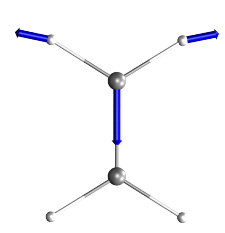

4

$1 b_{3 u}$

Figure 7: Scaled vector representation of $\Phi_{\alpha}^{i}$ for the five highest energy occupied canonical MOs of ethene at the HF/aug-cc-pVQZ level. Note that only half of the atoms have their vector drawn, the other half would be analogous as per the molecular symmetry, and have not been reproduced for clarity.

Thus, performing DOF analysis, at least in valence MOs with large basis sets, appears to be valid in both the HFT and the KS-DFT contexts. This fact has to be understood pragmatically: Koopmans' theorem is usable due to error cancellation between the lack of correlation and the frozen orbital approximation. Empirically, we have shown that, presumably through error cancellation as well, this methodology works well with KS-DFT orbitals.

This does not ensure, by any means, that DOF estimate the true, exact cationic gradient, as in all cases we are limited by the precision of the calculation. The inclusion of core-valence functions might be beneficial for the correct description of core-hole states, and yet not be flexible enough to capture the variations in the amplitudes of MOs. Diffuse functions seem to improve the description of $\Phi_{\alpha}^{i}$ without compromising the core-hole states significantly, and are therefore recommended in DFT calculations if the DOF analysis framework is to be used.

In the following sections, two brief example applications will be detailed using these established guidelines.

\section{Example application I: aromatic and antiaromatic molecules}

Two small conjugated systems, benzene, cyclobutadiene, have been examined using DOFs to exemplify the usefulness of the technique. From the results of the previous sections, an aug-cc-pVDZ basis set with the PBE0 DFA was deemed acceptable for the calculations in 
this section.

In the case of the benzene-cyclobutadiene pair, the question that will be investigated is whether aromaticity and antiaromaticity can be characterized by the response of $\pi$ orbitals to deformation. A similar procedure had been used in the past to investigate $\sigma$ and $\pi$ contributions to symmetry. ${ }^{33}$ In our case, the set of $\Phi_{\alpha}^{i}$ was calculated for the occupied MOs of both molecules. The initial expectation is that the $\pi_{1}, \pi_{2}$ and $\pi_{3}$ MOs of benzene are bonding, while the $\pi_{2}$ and $\pi_{3}$ MOs of cyclobutadiene are fundamentally nonbonding due to the perpendicular nodal planes. These two well-known arguments can be derived from simple Frost circles based on Hückel's rule (Fig. 8).
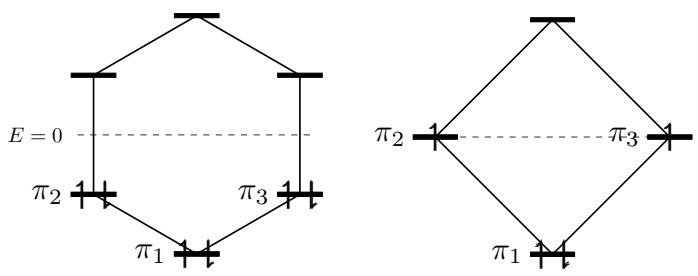

Figure 8: Frost circles for bencene and cyclobutadiene showing the qualitative nature of the $\pi$ MOs.

Note that in this case obtaining the HOMO cations for these two species is in principle doable, but accessing specific HOMO- $n$ cations is extremely difficult due to near-degeneracy. In our case, for exemplary purposes, an open-shell singlet configuration with $D_{4 h}$ symmetry was imposed for cyclobutadiene for illustrative purposes. 

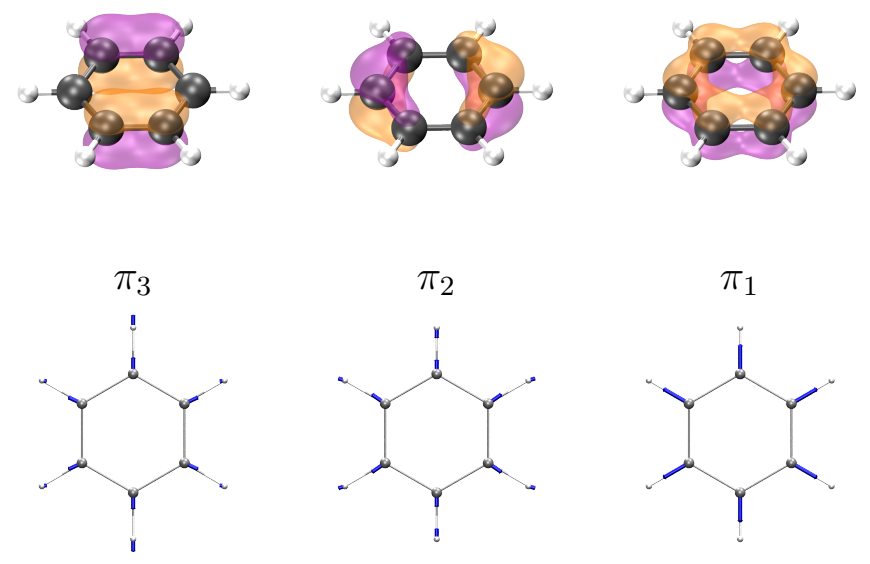

Figure 9: Isosurface representation of the three highest energy occupied canonical MOs of $\pi$ symmetry of benzene (top) and their corresponding set of $\Phi_{\alpha}^{i}$ (bottom).
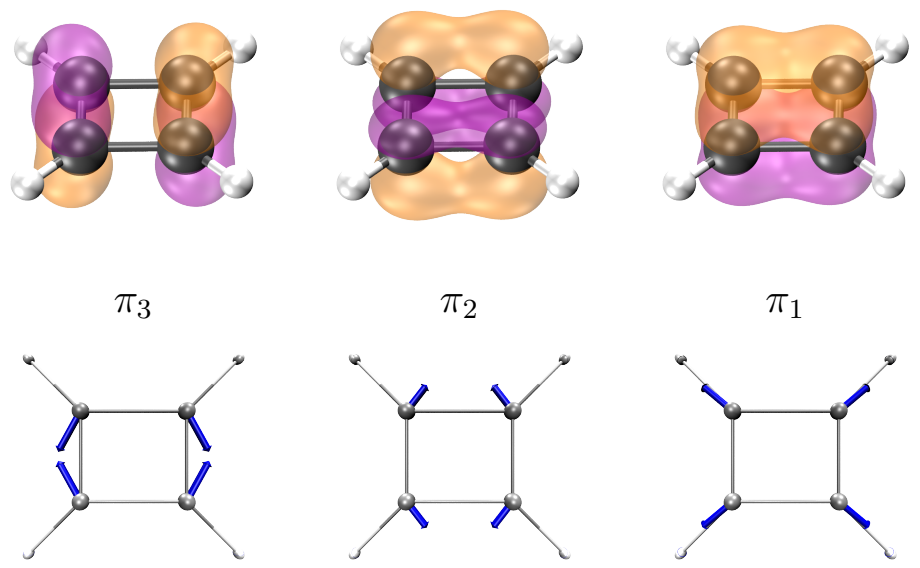

Figure 10: Isosurface representation of the three highest energy occupied canonical MOs of $\pi$ symmetry of cyclobutadiene (top) and their corresponding set of $\Phi_{\alpha}^{i}$ (bottom).

MOs for both species are represented in the top section of Figures 9 and 10, with their corresponding $\Phi_{\alpha}^{i}$ forces in proportional vectorial representation depicted below. As it can be clearly seen by examining the direction of the induced forces, the assumptions made previously are accurately captured by our approach. The forces arising from the HOMO and HOMO-1 pair $\pi_{2}$ and $\pi_{3}$ in benzene are small, but undoubtedly pull the carbon ring closer. The $\pi_{1}$ orbital has a significantly stronger binding force, which is in agreement with its much more stable and binding nature.

In the case of cyclobutadiene, the highest singly occupied MOs, $\pi_{2}$ and $\pi_{3}$, are shown to 
be bonding for the two possible double bond configurations, but not binding for the molecule altogether: electron removal from either will favor fragmentation. Only the $\pi_{1}$ contributes to global molecular binding, analogous to the one in benzene. As a small precision, note that the forces depicted for the $\pi_{2}$ and $\pi_{3}$ MOs are not exactly the same due to differences between what should be two perfectly degenerate MOs. This is a limitation of single-determinant frameworks, and not an issue that arises from DOF analysis. In fact, averaging over the supposedly degenerate pairs in both systems clarifies that the HOMO is clearly bonding for benzene (the forces add up), and fundamentally nonbonding in cyclobutadiene (the forces cancel out). Insomuch as the DFT calculations are accurate enough, our analysis is in agreement with the classic results from Hückel's rule.

\section{Example application II: characterization of MOs of acrolein}

Acrolein (2-propenal) is another simple molecule in which the $\pi$ system is highly important. In this case constructing the MO diagram and deriving conclusions from it is slightly more challenging, partly because of the symmetry loss that the oxygen atom exerts. Nevertheless, the excited state chemistry of acrolein is very relevant and has been studied very thoroughly over the years from the experimental and theoretical points of view. ${ }^{34-36}$

In this case, $\Phi_{\alpha}^{i}$ have been calculated for all the occupied MOs of the $s$-trans conformer at the PBE0/aug-cc-pvdz level, in an attempt to classify each valence MO completely ab initio. The highest energy occupied MOs are presented in Figure 11 as isosurfaces. As previously stated, predicting the role of each MO by examining the isosurfaces is increasingly difficult as they grow more delocalized with molecular size. Furthermore, the HOMO-1, HOMO-2 and HOMO-3 are all relatively close in energy which complicates the spectroscopic study of excitations from those levels.

Results are captured in Figure 12. According to the calculated DOFs, orbital 15 (the HOMO) is fundamentally nonbonding, with no forces being exerted on nuclei. MO 14 is strongly bonding for the $\mathrm{C}=\mathrm{C}$ bond, as suggested by its depiction. MO 13, however, is strongly antibonding for that very same bond. MO 12 is strongly bonding for the $\mathrm{C}=\mathrm{O}$ bond, while MO 11 (HOMO-4) is bonding for both double bonds, albeit less-so. According to this analysis, excitations arising from MO 14 should significantly weaken the $\mathrm{C}=\mathrm{C}$ bond, 
facilitating torsion, and excitations from MO 12 should weaken the $\mathrm{C}=\mathrm{O}$ bond enormously. However, excitation from MO 15 should not weaken the bonds in the molecule per se, and electron removal from MO 13 should in fact make the $\mathrm{C}=\mathrm{C}$ bond stronger and thus harder to rotate. Such detailed ab initio characterization is not possible through other techniques at a negligible cost.

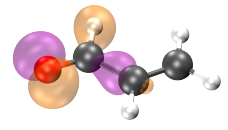

15

$13 a^{\prime}$

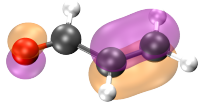

14

$2 a^{\prime \prime}$

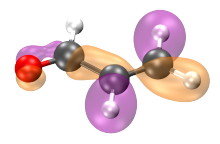

13

$12 a^{\prime}$

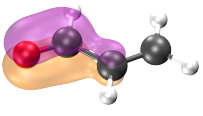

12

$1 a^{\prime \prime}$

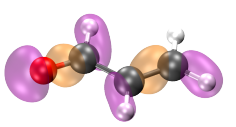

11

$11 a^{\prime}$

Figure 11: Isosurface representation of the five highest energy occupied canonical MOs of acrolein and their respective symmetry labels.

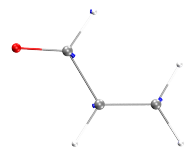

15

$13 a^{\prime}$

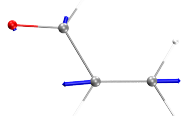

14

$2 a^{\prime \prime}$

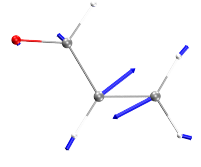

13

$12 a^{\prime}$

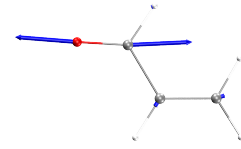

12

$1 a^{\prime \prime}$

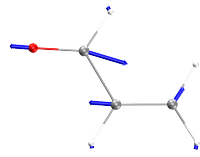

11

$11 a^{\prime}$

Figure 12: Scaled vector representation of $\Phi_{\alpha}^{i}$ for the five highest energy occupied canonical MOs of acrolein at the PBE0/aug-cc-pVDZ level.

Therefore, the study of DOFs can be of interest, too, when studying excited state chemistry. The interpretation of MO response to excitation can be complemented with DOF analysis, which requires no additional knowledge and is relatively inexpensive. For instance, coupling of DOF analysis with active space elucidation might be a future possibility for future development.

\section{CONCLUSIONS}

Dynamic Orbital Forces, which are originally derived from Koopmans' theorem, have been shown to be a generalized case of Nuclear Fukui Functions. Several interpretations have 
been given for such forces, which can be calculated as the opposite of the derivatives of orbital eigenvalues with respect to nuclear displacement. The validity of such forces has been empirically tested for both HFT and DFT, and shown to be consistent for valence orbitals as long as a diffuse basis set is used. Core orbital response can not be accurately captured due to massive orbital relaxation effects.

The quality of results is at least qualitative, and in many cases can be expected to be quantitative. Therefore, DOFs may be used to unequivocally classify valence MOs as bonding, nonbonding or antibonding in any system that can be reasonably described using a single-determinantal KS-DFT approach. However, as with everything in the Kohn-Sham DFT framework, the errors introduced by the approximate functional, along with inherent errors from the $\Delta$-SCF procedure, may hamper the process, and caution is always advised.

For interpretative purposes, vectorial representations are a simple way to analyze the effect of occupation of different MOs, which provides immediate insight for excited states calculations and a way to rationalize molecular structure and properties (e.g. aromaticity and antiaromaticity). The coupling of DOFs with orbital localization schemes is a promising future development. A formalism that can be reliably extended to virtual orbitals would allow for additional applications in excited state chemistry.

\section{ACKNOWLEDGMENTS}

RL acknowledges the LabEx MiChem program of Sorbonne Université for a mobility grant, ECOS-Sud action C17E09 for financial support, and ED388 for a PhD grant. CC acknowledges support by FONDECYT through project No. 1181121, and also by Centers Of Excellence With Basal/Conicyt Financing, Grant FB0807. PWA thanks NSERC, Compute Canada and the Canada Research Chairs. 


\section{References}

1. P. Chaquin and F. Fuster, ChemPhysChem 18, 2873 (2017), https://onlinelibrary.wiley.com/doi/pdf/10.1002/cphc.201700820, URL https://onlinelibrary.wiley.com/doi/abs/10.1002/cphc. 201700820.

2. P. Chaquin, Y. Canac, C. Lepetit, D. Zargarian, and R. Chauvin, International Journal of Quantum Chemistry 116, 1285 (2016), https://onlinelibrary.wiley.com/doi/pdf/10.1002/qua.25174,

URL https://onlinelibrary.wiley.com/doi/abs/10.1002/qua. 25174.

3. P. Chaquin, F. Fuster, and F. Volatron, International Journal of Quantum Chemistry 118, e25658 (2018), https://onlinelibrary.wiley.com/doi/pdf/10.1002/qua.25658, URL https://onlinelibrary.wiley.com/doi/abs/10.1002/qua. 25658.

4. F. Fuster and P. Chaquin, International Journal of Quantum Chemistry 119, e25996 (2019), https://onlinelibrary.wiley.com/doi/pdf/10.1002/qua.25996, URL https://onlinelibrary.wiley.com/doi/abs/10.1002/qua. 25996.

5. P. J. Robinson and A. N. Alexandrova, The Journal of Physical Chemistry A 119, 12862 (2015), pMID: 26616443, https://doi.org/10.1021/acs.jpca.5b09687, URL https://doi.org/10.1021/acs.jpca.5b09687.

6. Y. Tal and J. Katriel, Theoretica Chimica Acta 46, 173 (1977), URL https://doi.org/10.1007/bf00578227.

7. S. McKechnie, G. H. Booth, A. J. Cohen, and J. M. Cole, The Journal of Chemical Physics 142, 194114 (2015), https://doi.org/10.1063/1.4921037, URL https://doi.org/10.1063/1.4921037.

8. G. Zhang and C. B. Musgrave, The Journal of Physical Chemistry A 111, 1554 (2007), pMID: 17279730, https://doi.org/10.1021/jp061633o, URL https://doi.org/10.1021/jp061633o. 
9. T. Tsuneda, J.-W. Song, S. Suzuki, and K. Hirao, The Journal of Chemical Physics 133, 174101 (2010), https://doi.org/10.1063/1.3491272, URL https://doi.org/10.1063/1.3491272.

10. R. van Meer, O. V. Gritsenko, and E. J. Baerends, Journal of Chemical Theory and Computation 10, 4432 (2014), pMID: 26588140, https://doi.org/10.1021/ct500727c, URL https://doi.org/10.1021/ct500727c.

11. N. Pueyo Bellafont, F. Illas, and P. S. Bagus, Phys. Chem. Chem. Phys. 17, 4015 (2015), URL http://dx.doi.org/10.1039/C4CP05434B.

12. N. Colonna, N. L. Nguyen, A. Ferretti, and N. Marzari, Journal of Chemical Theory and Computation 15, 1905 (2019), pMID: 30640457, https://doi.org/10.1021/acs.jctc.8b00976, URL https://doi.org/10.1021/acs.jctc.8b00976.

13. R. M. Parrish, L. A. Burns, D. G. A. Smith, A. C. Simmonett, A. E. DePrince, E. G. Hohenstein, U. Bozkaya, A. Y. Sokolov, R. Di Remigio, R. M. Richard, et al., Journal of Chemical Theory and Computation 13, 3185 (2017), pMID: 28489372, https://doi.org/10.1021/acs.jctc.7b00174, URL https://doi.org/10.1021/acs.jctc.7b00174.

14. A. T. B. Gilbert, N. A. Besley, and P. M. W. Gill, The Journal of Physical Chemistry A 112, 13164 (2008), pMID: 18729344, https://doi.org/10.1021/jp801738f, URL https://doi.org/10.1021/jp801738f.

15. G. M. J. Barca, A. T. B. Gilbert, and P. M. W. Gill, Journal of Chemical Theory and Computation 14, 1501 (2018), pMID: 29444408, https://doi.org/10.1021/acs.jctc.7b00994, URL https://doi.org/10.1021/acs.jctc.7b00994.

16. T. Verstraelen, P. Tecmer, F. Heidar-Zadeh, C. E. Gonzlez-Espinoza, M. Chan, T. D. Kim, K. Boguslawski, S. Fias, S. Vandenbrande, D. Berrocal, et al., Horton 2.1.1, http://theochem.github.com/horton/ (2017-2020). 
17. Q. Sun, T. C. Berkelbach, N. S. Blunt, G. H. Booth, S. Guo, Z. Li, J. Liu, J. D. McClain, E. R. Sayfutyarova, S. Sharma, et al., Wiley Interdisciplinary Reviews: Computational Molecular Science 8, e1340 (2018), https://onlinelibrary.wiley.com/doi/pdf/10.1002/wcms . 1340,

URL https://onlinelibrary.wiley.com/doi/abs/10.1002/wcms. 1340.

18. Q. Sun, Journal of Computational Chemistry 36, 1664 (2015), https://onlinelibrary.wiley.com/doi/pdf/10.1002/jcc.23981,

URL https://onlinelibrary.wiley.com/doi/abs/10.1002/jcc.23981.

19. M. A. Marques, M. J. Oliveira, and T. Burnus, Computer Physics Communications 183, 2272 (2012), ISSN 0010-4655, URL http://www.sciencedirect.com/science/article/pii/S0010465512001750.

20. W. Humphrey, A. Dalke, and K. Schulten, Journal of Molecular Graphics 14, 33 (1996).

21. F. W. Averill and G. S. Painter, Phys. Rev. B 34, 2088 (1986), URL https://link.aps.org/doi/10.1103/PhysRevB.34.2088.

22. M. H. Cohen, M. V. Ganduglia-Pirovano, and J. Kudrnovský, The Journal of Chemical Physics 101, 8988 (1994), https://doi.org/10.1063/1.468026, URL https://doi .org/10.1063/1.468026.

23. M. H. Cohen, M. V. Ganduglia-Pirovano, and J. Kudrnovský, The Journal of Chemical Physics 103, 3543 (1995), https://doi.org/10.1063/1.470238, URL https://doi.org/10.1063/1.470238.

24. B. G. Baekelandt, The Journal of Chemical Physics 105, 4664 (1996), https://doi.org/10.1063/1.472329, URL https://doi.org/10.1063/1.472329.

25. R. Balawender, F. De Proft, and P. Geerlings, The Journal of Chemical Physics 114, 4441 (2001), https://aip.scitation.org/doi/pdf/10.1063/1.1346579, URL https://aip.scitation.org/doi/abs/10.1063/1.1346579. 
26. R. Balawender and P. Geerlings, The Journal of Chemical Physics 114, 682 (2001), https://aip.scitation.org/doi/pdf/10.1063/1.1331359, URL https://aip.scitation.org/doi/abs/10.1063/1.1331359.

27. J. F. Janak, Phys. Rev. B 18, 7165 (1978), URL https://link.aps.org/doi/10.1103/PhysRevB.18.7165.

28. E. J. Baerends, Molecular Physics 118, e1612955 (2020), https://doi.org/10.1080/00268976.2019.1612955, URL https://doi.org/10.1080/00268976.2019.1612955.

29. L. J. Bartolotti and P. W. Ayers, The Journal of Physical Chemistry A 109, 1146 (2005), pMID: 16833424, https://doi.org/10.1021/jp0462207, URL https://doi.org/10.1021/jp0462207.

30. T. H. Dunning, The Journal of Chemical Physics 90, 1007 (1989), https://doi.org/10.1063/1.456153, URL https://doi.org/10.1063/1.456153.

31. D. E. Woon and T. H. Dunning, The Journal of Chemical Physics 103, 4572 (1995), https://doi.org/10.1063/1.470645, URL https://doi.org/10.1063/1.470645.

32. R. A. Kendall, T. H. Dunning, and R. J. Harrison, The Journal of Chemical Physics 96, 6796 (1992), https://doi.org/10.1063/1.462569, URL https://doi.org/10.1063/1.462569.

33. A. Gobbi, Y. Yamaguchi, G. Frenking, and H. F. Schaefer, Chemical Physics Letters 244, 27 (1995), URL https://doi.org/10.1016/0009-2614(95)00922-q.

34. M. Reguero, M. Olivucci, F. Bernardi, and M. A. Robb, Journal of the American Chemical Society 116, 2103 (1994), https://doi.org/10.1021/ja00084a056, URL https://doi.org/10.1021/ja00084a056.

35. D. Duflot, J.-P. Flament, I. C. Walker, J. Heinesch, and M.-J. Hubin-Franskin, The Journal of Chemical Physics 118, 1137 (2003), https://doi.org/10.1063/1.1527924, URL https://doi.org/10.1063/1.1527924. 
36. S. Mai, T. Müller, F. Plasser, P. Marquetand, H. Lischka, and L. González, The Journal of Chemical Physics 141, 074105 (2014), URL https://doi.org/10.1063/1.4892060. 